Proceedings of the 11th Polish-Japanese Joint Seminar on Micro and Nano Analysis, Gniew, September 11-14, 2016

\title{
Nanoscale Analysis of Superparamagnetic Systems
}

\author{
A. Chrobak* And G. ZióŁKowski \\ Institute of Physics, Silesian University, Uniwersytecka 4, 40-007 Katowice, Poland
}

\begin{abstract}
The paper refers to a kind of nanoscale characterization of superparamagnetic materials above and below the so-called blocking temperature. It is propose to apply the Stoner-Wohlfarth model of nanoparticles magnetization supplemented by the two-level kinetic model, determining behavior in $T>0$. This approach allows determination of distribution of magnetic moments and energetic barriers of magnetic objects based on magnetization curves. In many cases, the determined distribution of magnetic moments can be recalculated into average size of the magnetic objects giving finally a nano/microscaled picture of the material. The proposed method was successfully used in characterization of diluted magnetics, nanocomposites, powders and even for human hemoglobin. In the paper the basic theory and its application to the nanoscale characterization is discussed in detail.
\end{abstract}

DOI: 10.12693/APhysPolA.131.1371

PACS/topics: 81.07.Bc, 75.50.Tt, 75.60.-d, 76.80.+y

\section{Introduction}

Nowadays, nanotechnology is a key point for materials development, including modern magnetic materials with superb parameters required for many applications. Such materials usually contain interacting or non-interacting magnetic objects like grains, particles, or powders. In the case of non-interacting systems, one can expect superparamagnetic properties with a characteristic magnetic moment and magnetic anisotropy (important below the so-called blocking temperature). The paper refers to a kind of nanoscale imaging of superparamagnetic materials in the both ranges - above and below the blocking temperature, i.e. determination of magnetic moments distribution based on empirical magnetization curves [13]. Such characterization can be helpful in the case of magnetic nanocomposites, diluted magnets, magnetic nanopowders as well as other materials containing magnetic nano/micro-objects. Moreover, in real materials a size distribution of magnetic grains (objects) is also expected, therefore, a determination of magnetic moments distribution can lead to a size imaging of the objects. The problem is not a trivial one. Relatively large ferro/ferrimagnetic particles behave differently below and above the blocking temperature $T_{b}$, due to the magnetic anisotropy. Especially in temperatures below $T_{b}$ it is necessary to include into considerations some energy barrier caused by the anisotropy.

During last years we have developed numerical procedures and models used for such analysis $[4,5]$. The aim of this paper is a consistent presentation of our approach which can be applied not only in the case of determination of magnetic moments distribution but also in similar problems in which thermal activation plays an important role. The proposed method was successfully used in characterization of diluted magnetics (alloys [6] and natural

*corresponding author; e-mail: artur.chrobak@us.edu.pl samples [7]), nanocomposites, powders and even for human hemoglobin [8]. The presented approach, with some restriction, can be also applied to the systems containing interacting magnetic objects [9].

\section{Theoretical background}

In some cases, magnetization process can be considered as coherent rotation of atomic magnetic moments. In a vicinity of the magnetic anisotropy, such precess should be analyzed as a kind of competition between different energies, i.e. magnetostatic energy, anisotropy energy, and thermal energy, leading to lowering of total free energy of the system. Magnetostatic energy $\mu_{0} \boldsymbol{H} \cdot \boldsymbol{\mu}$, interaction between magnetic moment $\boldsymbol{\mu}$ and external magnetic field $\boldsymbol{H}$, prefers a parallel alignment of the $\boldsymbol{H}$ and $\boldsymbol{\mu}$ vectors. Anisotropy energy, for simplicity uniaxial one, can be expressed by its density equal to $K \sin ^{2}(\theta)$ where $K$ is the uniaxial magnetic anisotropy coefficient and $\theta$ is the angle between magnetic moment and the so-called easy magnetization axis. It is obvious that a change of magnetic moment direction from antiparallel to parallel alignment to the field requires a "jump" over energy barrier originating from the anisotropy and external magnetic field, as shown in Fig. 1. Without magnetic field, the energy barrier is caused only by magnetic anisotropy and it is symmetric ( $E_{b}$ in Fig. 1). In the vicinity of the external magnetic field the barrier is antisymmetric due to the difference between magnetostatic energy of the particle with magnetization aligned parallel and antiparallel to the field. Additionally, in temperatures higher than zero, spontaneous jumps over the barrier occur with the Boltzmann probability $\exp \left(E_{b} / k_{\mathrm{B}} T\right)$, where $E_{b}$ is the energy barrier, $k_{\mathrm{B}}$ is the Boltzmann constant and $T$ is the temperature.

Kinetics of such process can be described by the following equations:

$$
\begin{aligned}
& \frac{\mathrm{d} n_{X}}{\mathrm{~d} t}=W_{Y X} n_{Y}-W_{X Y} n_{X}, \\
& \frac{\mathrm{d} n_{Y}}{\mathrm{~d} t}=W_{X Y} n_{X}-W_{Y X} n_{Y},
\end{aligned}
$$

where $n_{X}$ is the number of objects (per volume unit) in 
antiparallel

parallel

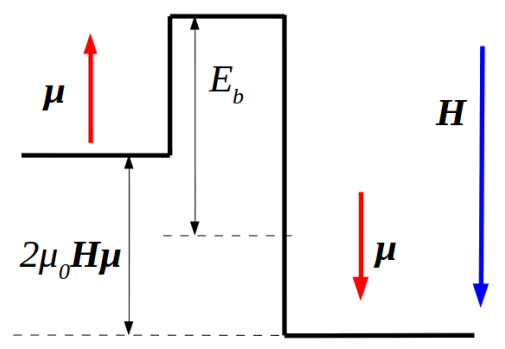

Fig. 1. Energetic diagram of the considered magnetization precess (see the text).

state $X$ (antiparallel), $n_{Y}$ is the number of objects (per volume unit) in state $Y$ (parallel), $W_{X Y}$ and $W_{Y X}$ is the transition frequency from $X$ to $Y$, and from $Y$ to $X$, respectively. The quantities $W_{X Y}$ and $W_{Y X}$ may be written as

$$
\begin{aligned}
& W_{X Y}=W_{0} \exp \left(-\frac{E_{b}-h}{k_{\mathrm{B}} T}\right), \\
& W_{Y X}=W_{0} \exp \left(-\frac{E_{b}+h}{k_{\mathrm{B}} T}\right),
\end{aligned}
$$

where $W_{0}$ is the jump frequency for $T=\infty$ and $h=$ $\mu_{0} \boldsymbol{H} \cdot \boldsymbol{\mu}$.

The set of Eqs. (1) and (2) have analytical exact solution allowing calculation a degree of filling of the two states $X$ and $Y$ of a population of magnetic moments as a function of external magnetic field as well as temperature. In order to perform simulations of hysteresis loops it is necessary to know the energy barrier regarding different position of the easy magnetization axis and the external field, only in the parallel case $E_{b}=K V$. For a system of non-interacting magnetic objects with different magnetic moments, anisotropy and space alignment one can determine the energy diagram (like in Fig. 1) directly using the Stoner-Wohlfarth model [10, 11] describing magnetization precesses of superparamagnetic particles at the ground state $(T=0)$. It should be underlined that the combination of the Stoner-Wohlfarth model and the twolevel energetic model allow to extend the first one in the temperatures higher than zero and, in a consequence, to model magnetic behavior of real superparamagnetic (non-interacting) systems.

Let us consider a material containing ferromagnetic non-interacting particles. Each particle is characterized by its total magnetic moment, uniaxial magnetic anisotropy, and space alignment of the easy magnetization axis. The whole material is described by distributions of the mentioned quantities. In practice, magnetization precess of the considered system can be analyzed as a balance between the particles that changed and not their magnetization direction over individual energy barrier. This approach leads to some indistinguishability of two kind on magnetic objects, let us say $\alpha$ and $\beta$, for which $E_{b}^{\alpha}-\mu_{0} \mu^{\alpha} H=E_{b}^{\beta}-\mu_{0} \mu^{\beta} H[5]$. Those objects can be activated with the same probability at a given temperature.
Furthermore, one can assume that for a typical measurement time $100 \mathrm{~s}$, without external magnetic field and at temperature $T$, all objects that satisfy the condition $E_{b}<25 k_{\mathrm{B}} T$ spontaneously change their magnetization over the barrier. The indistinguishability of magnetic objects is expressed by the line $25 k_{\mathrm{B}} T=E_{b}-\mu_{0} \mu H$ called $H$-line, for which the objects are equivalent. In $E_{b} \mu$ space, all objects with parameters below the $H$-line contribute to change of magnetization, see Fig. 2. As shown, the increase of external magnetic field, at a given temperature, causes the increase of the $H$-line slope which leads to progressive change of sample magnetization dependently on the distribution of magnetic object in $E_{b} \mu$ space. The highest magnetization change should be observed when the $H$-line passes a maximum of the magnetic cluster distribution (in our example - the central point).

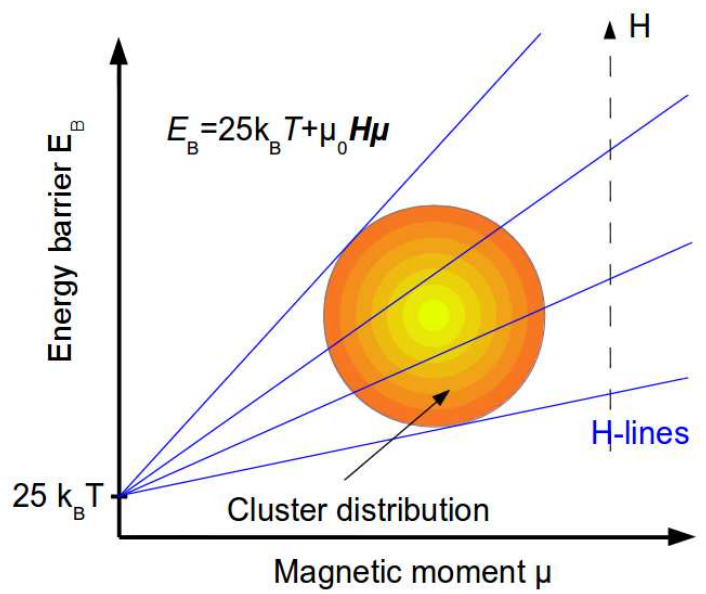

Fig. 2. Scheme of magnetization precess of a superparamagnetic system with the idea of the $H$-lines.

\section{Numerical analysis of superparamagnetic nanoscale systems}

The obvious question is: (i) for which materials the presented magnetization model can be applied and (ii) how the materials can be characterized using this model? First of all, the considered model of magnetization process is valid for magnetic materials composed of noninteracting magnetic moments or single domain particles. The single domain condition ensures coherent rotation of magnetic moment inside the particle. An answer for the second question is more complex. One can simulate magnetic characteristics based on a known system, i.e. known distribution in $E_{b} \mu$ space of the magnetic objects. The second possibility is to determine the object distribution based on empirical magnetization curves. Moreover, in the case when crystal structure of the particles is known, it is possible to rescale magnetic moment into size that is called the Langevin granulometry [12]. The critical point is if the system is blocked or unblocked, i.e. below or above the blocking temperature. For the unblocked state, the problem is quite easy but also requires 
applying sophisticated numerical methods, as described in [4]. Our development refers to the blocked superparamagnets in which energy barrier, related to the magnetic anisotropy, plays an important role. As it was mentioned, the indistinguishability, reflected by the $H$-lines, makes impossible determination of the magnetic moments distribution which is the main difficulty of applying the Langevin granulometry to the blocked systems. The idea is to "observe" the searched distribution in the $E_{b} \mu$ space by the $H$-lines, starting from two different temperatures, see Fig. 3.

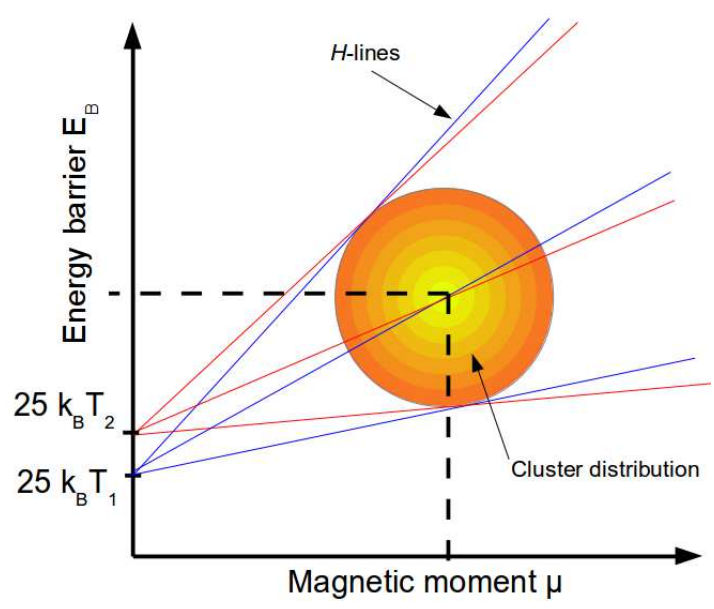

Fig. 3. The idea of determination of distribution homological points (see the text).

As can be seen, for the same homological distribution points (e.g. maximum), the determined at different temperatures $H$-lines (with different slopes) intersect, indicating real position in the $E_{b} \mu$ space. Assuming a Gaussian-like shape of the distribution and using simple geometrical relations one may calculate its expected value and width. Only one problem is how to determine the $H$-lines based on the $M(H)$ magnetization curves. For this purpose, the best are so-called remagnetization curves, i.e. after initial saturation in one direction remagnetization to saturation but in opposite direction. Generally, by fitting the model to such curves only a trace of the distribution can be determined, due to the indistinguishability along the $H$-lines. Furthermore, from the traces a profile of the distribution as a sum of objects along the $H$-lines with different slopes (or different $H$ ) is calculated. The profile reflects a shape of the real distribution, "visible" from the given temperature, exhibiting also the homological points. Finally, from the temperature shift of the profile maxima, the expected value of the distribution can be calculated. Such procedure is described in detail in [5]. Figure 4 shows exemplary results for the assumed Gaussian distribution of magnetic clusters with an expected value of maximum placed at $E_{b}=1 \mathrm{eV}$ and $\mu=5000 \mu_{\mathrm{B}}$. Briefly, for the assumed distribution the remagnetization curves were simulated for $T=300$ and $100 \mathrm{~K}$, next, the distribution traces and profiles were determined based on these curves. As shown, the recreated values of the distribution confirm correctness of our model and calculation procedure.

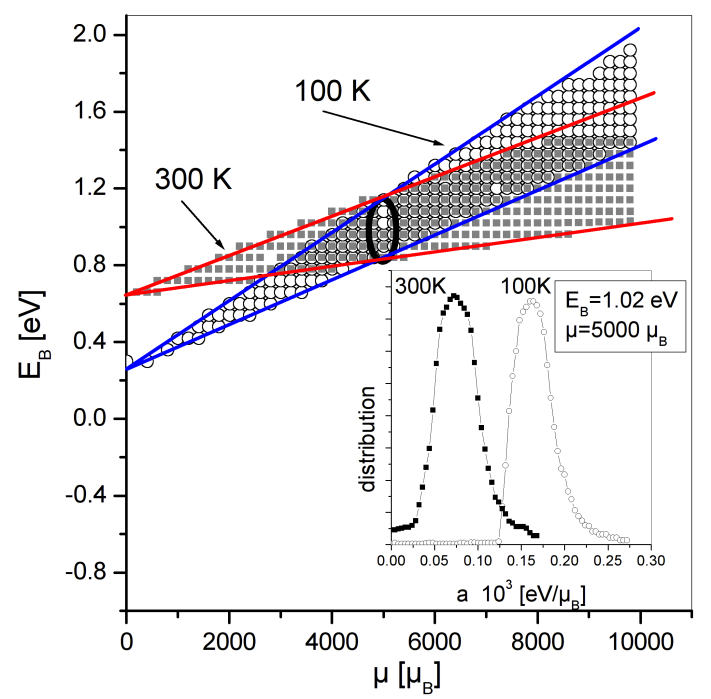

Fig. 4. Example of the distribution traces and profiles (in the inset) obtained for the assumed cluster distribution (the dark contour). The open and close points refer to a projection of distribution to the $E_{b} \mu$ plane independent of the intensity.

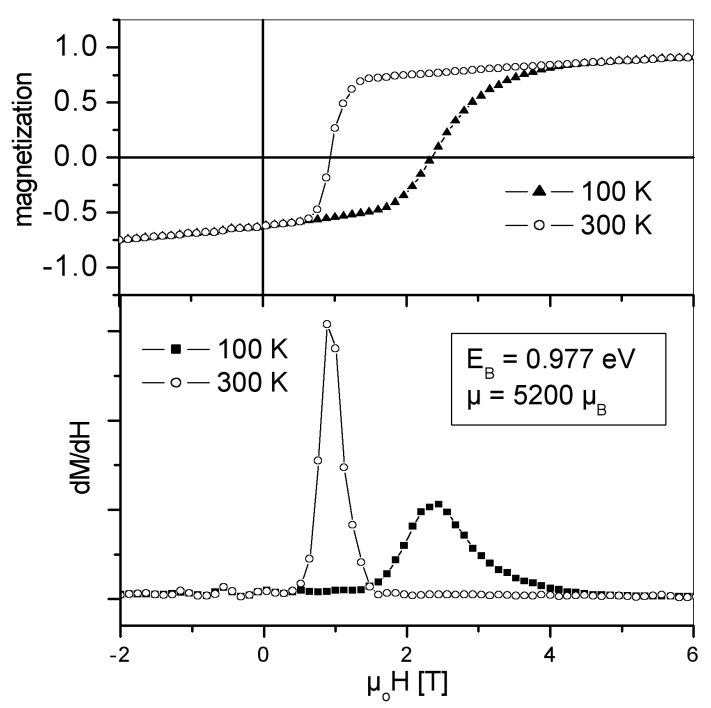

Fig. 5. Simulated remagnetization curves and their derivatives.

It should be stressed that the intercepts and ranges of slopes of the $H$-lines were obtained without any preliminary assumption about them. Moreover, the used numerical algorithm reveals the predicted indistinguishability.

In many cases, mean values of the searched cluster distribution is enough for characterization of studied materials. Here, some simplifications, allowing obtaining 
similar results without the usage of the specialized software, are possible. The maximum (or maxima) of the distribution profiles can be determined also from a simple derivative of the $M(H)$ remagnetization curves. Figure 5 shows an example of such analysis for the simulated $M(H)$ curves at two different temperatures $T=300$ and $100 \mathrm{~K}$, similarly to the case presented in Fig. 4.

As shown, the derivatives reveal well defined distribution's homological points as the maxima attributed to the highest magnetization change intensity. From a shift of the maxima determined at two different temperatures $T_{1}$ and $T_{2}$, one may calculate a real position of the distribution expected value, i.e. $\mu=25 k_{\mathrm{B}}\left(T_{2}-T_{1}\right) /\left(\mu_{0} H_{1}-\right.$ $\left.\mu_{0} H_{2}\right)$ and $E_{b}=25 k_{\mathrm{B}} T_{1}+\mu \mu_{0} H_{1}$, where $H_{1}$ and $H_{2}$ are positions of the maxima at $T_{1}$ and $T_{2}$, respectively. In our case $E_{b}=0.977 \mathrm{eV}$ and $\mu=5200 \mu_{\mathrm{B}}$ that quite well fit to the assumed values.

Let compare the two presented methods (see Fig. 4 and Fig. 5). The first one, using full analysis based on the Stoner-Wohlfarth and the two-level models, is more precise but requires specialized software. In the case when the analysis should give information about only a number of Gaussian distribution components and their mean values in the $E_{b} \mu$ space, the second simplified approach can be successfully used.

During last years the Langevin granulometry method was applied in our researches related to the both unblocked and blocked superparamagnetic systems. Impressive was analysis of $\mathrm{Ni}$ clusters in diluted magnets of $\mathrm{Al}-\mathrm{RE}-\mathrm{Ni}(\mathrm{RE}=\mathrm{Dy}, \mathrm{Gd})$ as a function of RE content [3]. It was shown that $\mathrm{Ni}$ forms small clusters containing about 300 atoms and with an increase of RE content the size of the clusters decreases to less than 100 . The performed numerical analysis, based on magnetic isotherms, allows showing and explaining the observed effect which was unique result of imaging of such small clusters. Also interesting was magnetic imaging of human blood specimens, coming from two kinds of patients: without and with atherosclerotic features [8]. Using the presented approach, it was shown heme-iron for the "healthy" samples and heme-iron plus its complex for the "sick" samples characteristic for this illness. Unfortunately, we tested only few samples, so diagnostic potential is not clear. However, correctness and usefulness of the method was strongly confirmed.

It was mentioned that the proposed method can be also used for the interacting particles, but with some reservations. First of all, the method is restricted to rather hard magnetic materials in which pinning mechanism is dominant in magnetization process. Accounting that the pinned magnetic objects are coupled with their surroundings, the analysis can reveal real magnetic moments and apparent energy barrier of pinning centers contributing to a change of magnetization. The apparent energy barrier is a quantity that includes not only magnetic anisotropy but also possible interactions with surroundings indicating energy required for the pinning. This approach was applied for $\mathrm{Fe}-\mathrm{Nb}-\mathrm{B}-\mathrm{Tb}$ bulk nanocrystalline alloys [9]. It was shown that the observed ultra-high coercivity (more than $7 \mathrm{~T}$ at room temperature) is caused by small pinning centers characterized by apparent energy barrier $1.2 \mathrm{eV}$ and mean magnetic moment $1000 \mu_{\mathrm{B}}$ which after rescaling means about $5 \mathrm{~nm}$ in size.

\section{Conclusions}

The aim of this paper was to collect and consistently describe the Langevin granulometry extension to the blocked state of superparamagnetic systems. In relation to the magnetic materials, in which the coherent rotation or pinning mechanism is dominant, one may formulate the following conclusions:

- The Stoner-Wohlfarth model can be extended to the temperatures higher than zero by the two-level energetic model.

- The Langevin granulometry method, supplemented by the presented approach, can be used in analysis of superparamagnetic systems below the blocking temperature.

- Determination of magnetic object distribution in the $E_{b} \mu$ space can be considered as additional nanoscale characterization method.

\section{Acknowledgments}

This work was supported by National Science Center in Poland by the grant 2015/19/B/ST8/02636.

\section{References}

[1] J.A. Potton, G.J. Daniell, A.D. Eastop, M. Kitching, D. Melville, S. Poslad, B.D. Rainford, H.J. Stanley, J. Magn. Magn. Mater. 39, 95 (1983).

[2] J. Abellan, R.M. Baker, F.P.A. Coolen, Eur. J. Oper. Res. 212, 112 (2011).

[3] P.A. Chernavskii, J.-A. Dalmon, N.S. Perov, A.Y. Khodakov, Oil Gas Sci. Technol. Rev. IFP 64, 25 (2009).

[4] A. Chrobak, G. Haneczok, G. Chełkowska, A. Kassiba, G. Ziółkowski, Phys. Status Solidi A 208, 2692 (2011).

[5] A. Chrobak, G. Haneczok, Phys. Status Solidi A 210, 1584 (2013)

[6] A. Chrobak, A. Slebarski, G. Haneczok, B. Kotur, J. Appl. Phys. 110, 113908 (2011).

[7] M.S. Bućko, O.-P. Mattila, A. Chrobak, G. Ziółkowski, B. Johanson, J. Cuda, J. Filip, R. Zboril, L.J. Pesonen, M. Lepparanta, Geophys. J. Int. 195, 159 (2013).

[8] B. Janus, M.S. Bucko, A. Chrobak, J. Wasilewski, M. Zych, J. Magn. Magn. Mater. 323, 479 (2011).

[9] A. Chrobak, G. Ziółkowski, N. Randrianantoandro, J. Klimontko, D. Chrobak, K. Prusik, J. Rak, Acta Mater. 98, 318 (2015).

[10] E.C. Stoner, E.P. Wohlfarth, Philos. Trans. R. Soc. A 240, 599 (1948), reprinted in, IEEE Trans. Magn. 27, 3475 (1991).

[11] A.P. Guimaraes, Principles of Nanomagnetism, Springer-Verlag, Berlin 2009.

[12] A. Kakay, M.W. Gutowski, L. Takacs, V. Franco, L.K. Varga, J. Phys. A 37, 6027 (2004). 